\title{
Video Streaming Evaluation of Path Efficient and Geographical Distance based AOMDV (PE-GD-AOMDV) Routing Protocol in MANET
}

\author{
Dr. M. Kokilamani ${ }^{1}$ \\ ${ }^{1}$ Assistant Professor, Department of Computer science, \\ Kamalam college of Arts and Science, Udumalpet-642126, Tamil Nadu, India. \\ [e-mail:kokilam.udt@gmail.com] \\ Dr. E. Karthikeyan ${ }^{2}$ \\ ${ }^{2}$ Head \& Assistant Professor, Department of Computer Science, \\ Government Arts College, Udumalpet-642126, Tamil Nadu, India. \\ [e-mail:e_karthi@yahoo.com] \\ ABSTRACT-
}

The demand of multimedia video streaming services in MANET is expected to significantly grow in the next years. Video streaming services require the provison of Quality of Service (QoS) and Quality of Experience (QoE) are the qualitative measures of the videos have to be delivered over wireless communication networks, but QoE reflects the user perception. In this paper, we proposed PE-GD-AOMDV protocol to increase the quality of video and made assessment by using EvalVid with NS2 framework to the quality of video transmission. PE-GD-AOMDV routing on H.264/MPEG-4 AVC video streaming is evaluated under both Distributed Coordination Function (DCF) and Enhanced Distributed Coordination Function (EDCF). Simulation results show that the PE-GD-AOMDV performs better than AOMDV with respect to PSNR and Throughput. Keywords: Multipath routing, MANET, QoS, QoE, AOMDV, PE-GD-AOMDV

Date of Acceptance: Apr 27, 2019

\section{Introduction}

I $n$ recent years, MANETs get a lot of attention from the research community because most important technical advances have arisen as a consequence. They are envisioned as an important type of access network of next generation, from which on-demand video streaming services are surely going to be increasing dramatically. MANETs are used in great variety of scenarios such as emergency rescue, conferences, universities, museums and exploration missions. These scenarios require the provision of QoS, which still remains an open issue in MANETs. However, communication in such environment is characterised by dynamic nature, limited bandwidth, high error rates, lack of centralized infrastructure and dynamic changes of wireless channel condition etc. Due to the mobility of wireless nodes, the established connection routes between senders and receivers are likely to be broken during video transmission, thus causing interruptions, freezes or jerkiness in the received video signal. These constraints and challenges, in combination with the delay and loss sensitive nature of video applications, made video communication over MANETs a challenging one [1][2].

Now-a-days H.264/MPEG-4 AVC Video Streaming delivery OoS and QoE [3][4] are expected to be an important component of many emerging multimedia applications. However, real-time video streaming is fundamentally different from traditional data communication. Recently H.264/MPEG-4 [5] is used to encode the video frames. The new H.264 provide enough flexibility to allow the standard to be applied to a wide variety of applications for both low and high bit rates, for low and high resolution video and with high and low demands on latency. The MPEG-4 video codec adds advanced error detection and correction services to the services that H.263. Even though some packet loss is tolerable, the quality of reconstructed video will be impaired and errors will propagate to consecutive video frames due to the dependency introduced among frames at the encoder. Therefore, the characteristics of mobile ad hoc real-time video streaming identify newly significant challenges for routing protocol.

Numerous multipath routing protocols get attention and are a promising technique for video delivery in MANET. Recent research demonstrates that it supports reimbursement such as robustness, load balancing, energy efficiency and increased throughput and so on. Thus, the application of multipath routing in wireless video streaming seems natural. However, few special characteristics of existing multipath routing protocols degrade the performance of video delivery. Hence, most of them just adapt to data communication without considering the characteristic of video steaming communication. As a result, it is desirable to modify multipath routing schemes to provide efficient video delivery.

In this work, PE-GD-AOMDV protocol is proposed for video streaming in MANET, including a series of novel routing mechanisms. The proposed scheme considers the characteristics of video communication to reduce delay and buffer time by means of introducing path selection metrics such as Active Path Indicator and 
Transmission distance, which reduce congestion, energy consumption, signal decay and mobility problems. To fix these problems to explore the cross-layer design framework for mapping the video frame parameters to the network layer. These video frames are encoded and evaluated by using EvalVid with NS2 framework to assess the quality of video transmission. Through a series of simulation experiments, it determines that the proposed PE-GD-AOMDV approach perform well with respect to packet/frame loss rate, packet/frame jitter, PSNR, and MOS metrics for video quality assessment purposes.

\subsection{Overview of EvalVid}

Publicly available tools for video quality evaluation often assume synchronized frames at the sender and the receiver side, which means they can't calculate the video quality in the case of frame drops or frame decoding errors. EvalVid is a framework and a toolkit for a unified assessment of the quality of video transmission. Enhanced EvalVid[6][7][8] is an extended framework and tool-set of EvalVid [9][10][11] [12] for evaluating the quality of the H.264/MPEG-4 AVC video transmitted over a real or simulated communication network. This framework provides packet/frame loss rate, packet/frame jitter, PSNR, and MOS metrics for video quality assessment purposes. The structure of the enhanced EvalVid framework is shown in Figure 1. The main components of the enhanced EvalVid are described as follows:

Source: The video source can be either in the YUV QCIF $(176 \times 144)$ or in the YUV CIF $(352 \times 288)$ formats.

Video Encoder and Video Decoder: Currently, EvalVid only supports single layer video coding. It supports three kinds of MPEG4 codecs, namely the NCTU codec, ffmpeg, and Xvid. The focus of this investigation is NCTU codec for video coding purposes.

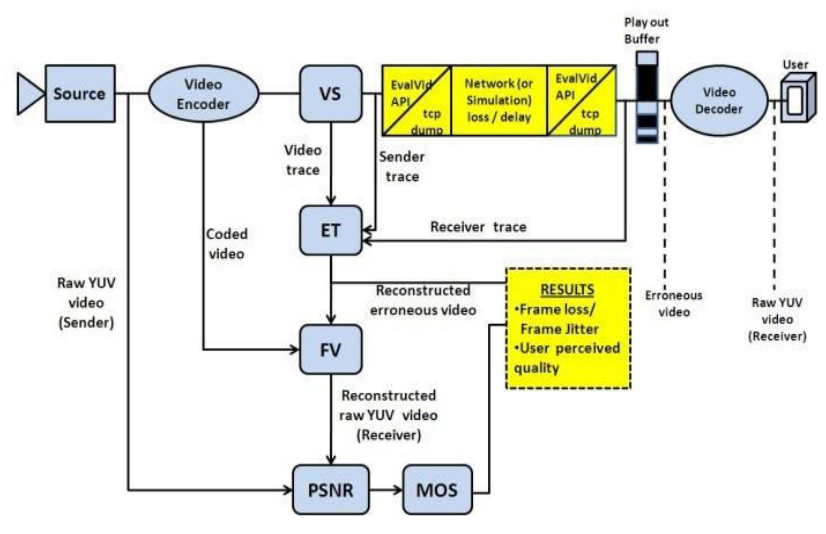

Fig. 1: Structure of enhanced EvalVid framework [13]

VS (Video Sender) - This component is used to read the compressed video file from the output of the video encoder, each large video frame is fragmented into smaller segments, and then transmitted these segments via UDP packets over a real or simulated network. This framework records the timestamp, the packet ID, and the packet payload size of each transmitted UDP packet in the sender trace file with the help of third-party tools such as tcp-dump [13] or win-dump [14] if the network is a real link.

ET (Evaluate Trace) - Once the video transmission is over, the information about the timestamp, the packet ID and the packet payload size available at the receiver has to be transported back to the sender in order to begin the evaluation task at the sender side. The ET component creates a frame/packet loss and frame/packet jitter report and generates a reconstructed video file based on the original encoded video file, the video trace file, the sender trace file and the receiver trace file which corresponds to the possibly corrupted video found at the receiver side as it would be reproduced to an end user.

FV (Fix Video) - Digital video quality assessment is performed frame by frame. Therefore, the total number of video frames at the receiver side including the erroneous frames must be the same as that of the original video at the sender side. If the codec cannot handle missing frames, the FV component is used to tackle this problem by inserting the last successfully decoded frame in the place of each lost frame as an error concealment technique [15].

\subsection{Integrated structure of enhanced EvalVid framework with NS2}

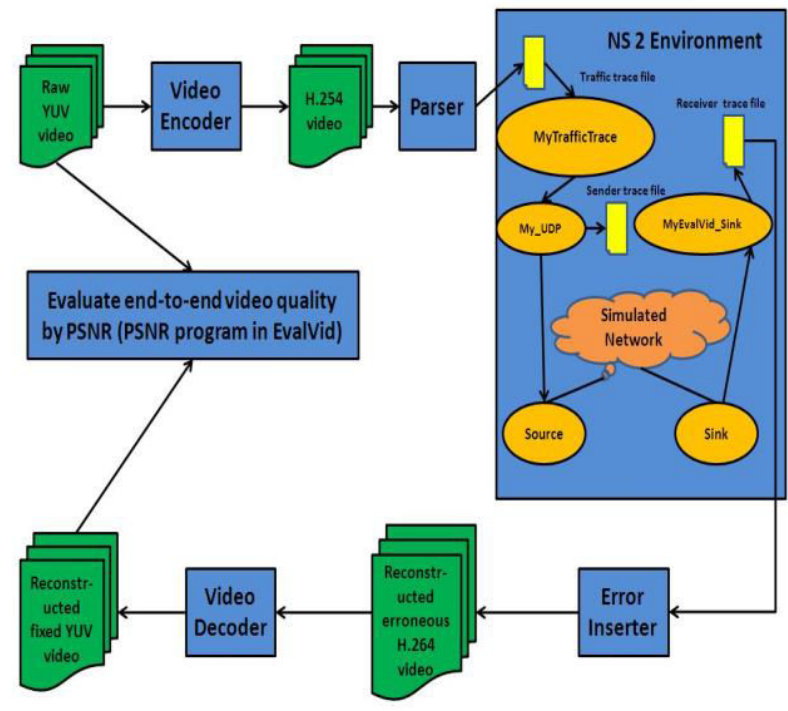

Fig. 2: Integrated structure of the enhanced EvalVid with ns-2 [13]

Figure 2. illustrates the integrated structure of enhanced EvalVid with NS2. The enhanced EvalVid has the following three interface programs for communicating with NS2: MyTrafficTrace, My_UDP, and MyEvalvid_Sink: (i) MyTrafficTrace agent extracts the frame type and the frame size of the video trace file generated from the traffic trace file, fragments the video frames into smaller segments, and sends these segments to the lower UDP layer at the appropriate time according to the user settings specified in the simulation script file. (ii) $M y_{-} U D P$ is an extension of the UDP agent that allows the 
user to specify the output file that is the name of the sender trace file which records the timestamp of each transmitted packet, the packet ID, and the packet payload size. The task of the My_UDP agent corresponds to the task that tools such as tcp-dump or win-dump performs in a real network environment. (iii) MyEvalvid_Sink is the receiving agent for the fragmented video frame packets sent by My_UDP. This agent also records the timestamp, packet ID, and payload size of each received packet which have been kept in the user specified receiver trace file.

As a result, the whole evaluation process starts from encoding the raw YUV video, and then the VS program will read the compressed file and generate the traffic trace file. After simulation, the ET program produces the corrupted video file based on these three trace files and the original encoded video. Subsequently, the corrupted video is decoded and error concealed. Finally, the reconstructed fixed YUV video can be compared with the original raw YUV video to evaluate the end-to-end delivered video quality.

The remainder of this paper is organized as follows. In Section, 2 we review the previous work and the basics of the EvalVid is described to analysis the video quality. Section 3 explains existing routing protocols and Section 4 presents proposed routing protocols in detail. Section 5 involves evaluation and experimental results. Finally Section 6 concludes the paper and some perspective for future work are outlined as well.

\section{Related Work}

M. A. Igartua et al [16] proposed a multipath routing technique. In this technique, initially routes are discovered by using dynamic source routing and a probe message packet is transmitted from source to destination using these routes. Once the first probe message is received at the destination end, a time out is triggered. The probe message reply packet is sent to the source using the same path from which it is received. Routes are classified as best route, medium route and worst route. Various paths are used for sending data according to data priorities. Best path is used for sending high priority packets, medium path is used for transmitting medium priority packets and worst path is used for sending least priority packets.

Dalei $\mathrm{Wu}$ et al. [17] proposed an application-centric routing framework for realtime video transmission in multihop wireless networks. They designed application-centric cross layer strategy to solve multi-hop routing problem. This approach minimizes end-to-end delay at the receiving end by computing an optimized routing path. Within the proposed quality-driven framework, video source coding has been integrated into the path routing to enhance the feasibility of multi-hop routing

Sonal Mishra and Anil Singh [18] proposed a new strategy for video transmission in MANET. The approach minimizes delay jitter problem by using cross layer approach from the integrationof MAC, network and transport layer. In MAC layer, the traffic classifer classifies the data and form a queue. At network layer predefined threshold is declared and compared with SNR, that is provided by the MAC layer. If SNR is less thn predefined threshold, the route is selected. Also at the application layer Application Packet Rate Controller (APRC) is used to control the paccket flow. This process mininmizes congestion in the channel and reduce delay jitter.

Mohammed Halloush et.al [19] evaluated the performance of communicating scalable video over MANET. AODV,DSR and OLSR are the three routing protocols evaluated. In the first scenario, the effect of increasing the number of hops between soruce and destination was assessed. Performance results showed that AODV and DSR achieve better perfrormance than OLSR. In the second scenario, a grid topology with mobile nodes randomly located was evaluated. The effect of increasing the numbet of connections over which video is stramed was assessed. Performance results showed that OLSR could achieve better performance in terms of video quality even though it achieves lower packet-delivery rates.

Schierl et al.[20] presemted a scheme based on Raptor Fec that uses different sources for reliable meida stramingin MANET scenarios woth high route loss probabilty. Their evaluation is basedo on ns-2 simulation.

Sheltami [21] evaluates the performance of H.264 protocol using two routing protoocls: the Neighbor Aware Clusteheae (NAC) and the Dynamic Source Routing (DSR) protocols. The author shows that it is feasible to have video over MANETs within an average distance of 6 hops, and requiring $5.5 \mathrm{Mbps}$ on average.

Calafate et al.[22] propose a QoS framework for MANETs combining IEEE 802.11e technology, a multipath routing algorithm, and a distributed admision control algorithm. Their solution was tested via simulation, and Peak Signal-to-Noise Ratio results were obtained under different network congestion conditions.

Ee and Song[23] propose an effective cross layer optimized video streamming algorithm over multi-hop MANET. Their algorithm attempts to satisfy an end-to-end delay constraint, while maintaining packet loss rate within a tolerable range at the receiver.

A cache-based on demand multipath routing protocol [24] it sends packets on multiple paths simultaneously in a round-robin manner. This is suitable for storage space is available for caching packets, which is not a requirement in our proposed large scale networks.

Calafate et al. [25] propose a QoS framework for MANETs combining IEEE 802.11e technology, a multipath routing algorithm, and a distributed admission control algorithm. They obtained a Peak Signal-to-Noise Ratio results under different network congestion conditions. An effective cross layer optimized video streaming algorithm over multi-hop mobile ad hoc networks. Their algorithm attempts to satisfy an end-to-end delay constraint, while maintaining packet loss rate within a tolerable range at the receiver. 


\section{Overview of AOMDV, GD-AOMDV and PE-GD-AOMDV routing}

\subsection{AOMDV multipath protocol}

AOMDV (Ad hoc On-demand Multipath Distance Vector) $[26,27]$ routing protocol is a multipath extension to AODV protocol aims to find loop-free and link-disjoint multi-paths during the route discovery process. AOMDV uses advertised hop-count to guarantee the loop free feature. Advertised hop-count is defined as the maximum hop-count of the multiple paths to a destination node available at an intermediate node i. It ensures that alternate paths at every node are disjoint, therefore achieves path disjointness without using source routing. To support multipath routing, route tables in AOMDV contain a list of paths for each destination. All the paths to a destination have the same destination sequence number. Once a route advertisement with a higher sequence number is received, all routes with the old sequence number are removed. Two additional fields, hop count and last hop, are stored in the route entry to help address the problems of loop freedom, and path disjointness, respectively. Because the protocol implement multipath discovery, the loop freedom guarantee from AODV no longer holds. AOMDV address this issue as follows. The hop count field contains the length of the longest path for a particular destination sequence number, and is only initialized once, at the time of the first advertisement for that sequence number.

Hence, the hop count remains unchanged until a path for a higher destination sequence number is received. It follows that loop freedom is ensured as long as a node never advertises a route shorter than one already advertised, and never accepts a route longer than one already advertised. To ensure that paths in the route table are link-disjoint, a node discards a path advertisement that has either a common next hop or a common last hop as one already in the route table. It was observed that, as long as each node adheres to this rule, all paths for the same destination sequence number are guaranteed to be link-disjoint.

Node-disjoint paths can be obtained with an additional restriction that for a particular destination sequence number, every node always advertises the same designated path to other nodes. Route maintenance in AOMDV is similar to that in AODV. A RERR for a destination is generated when the last path to that destination fails. In AOMDV, advertised_hopcount replaces hopcount in AODV. A route_list replaces the nexthop, and essentially defines multiple next hops with respective hopcounts. However, all next hops still have the same destination sequence number.

\subsection{Geographical Distance Based Ad Hoc On-demand Multipath Distance Vector Routing Protocol}

Geographical Distance Based Ad Hoc On-demand Multipath distance Vector Routing Protocol (GD-AOMDV) [28] selects multiple paths based on the transmission distance and choose minimum transmission distance path for communication. In Route Discovery phase, the RREQ packet collect the distance information of all intermediate nodes till it reaches the destination. Then destination sorts all available paths with the consideration of transmission distance value. The reason for choosing the paths based on the geographical distance is that to reduce end-to-end delay, congestion and to reduce large queue of packets to move along the shortest path traffic.

In general path selection is basically done by selecting minimum number hops on the path (Shortest path). Some time the selection of hops may be few but the transmission distance is high. While selecting shortest path the center of the network become more congestion compared to the perimeter of the network and leads to path breakage, packet loss, node over usage. Another thing to be considered is that when choosing shortest path, the hop is usually present at the transmission edge because of which earlier outcome of the hop from the transmission edge happens. So that topology of the network changes dynamically. Even though by using distance metric for path selection in the proposed GD-AOMDV scheme, the number of hops may increase the delivery of packet to the destination, reduces delay and decrease energy consumption.

If paths are chosen based on the distance then the number of hops within the network coverage is high, so that if any moves out of the coverage an alternate nearby hop takes the path and energy required for transmission is also optimally maintained. Besides the traffic outcome of the hop from the network coverage is the most important thing to be considered for selecting paths because the waiting time for the packets along the path reduces considerably.

The distance between two nodes can be determined by the Euclidean distance is as follows in equation 3.1 and 3.2 :

$$
D_{P_{i}}=\sqrt{\left(S_{x i}-S_{x j}\right)^{2}+\left(S_{y i}-S_{y j}\right)^{\prime}}-3.1
$$

where $S_{i}$ and $S_{j}$ are nodes to find the distance $\nu$ of the path $\mathrm{P}_{\mathrm{i}}$. To find distance of multiple path on the network $\mathrm{P}_{\mathrm{n}}$ is as follows:

$$
D_{P_{n}}=\sum_{P_{i}}^{P^{n}}\left(D_{p}\right)
$$

From the above discovered paths our approach selects the path that has the minimum transmission distance in equation 3.3 , that is

$$
G D-A O M D V=\min \left(D_{P_{n}}-3.3\right.
$$

While using this approach the power consumption, signal strength and mobility are intently achieved. We analysis and evaluate the occasions when a sender can use this mechanism to improve the packet delivery and transmission bandwidth. 


\subsection{Path Efficient Load Balanced Ad-hoc on-demand Multipath Distance Vector routing protocol}

In this part, we propose an extension to AOMDV protocol in order to support certain mechanism and technique to improve its performance. The AOMDV protocol selects the route with the lower hop count to forward data. However, the less congestion routes can provide short end to end delay than routes providing lower hop count. To choose the less congestion routes, we need a new metric which allow source node to select the less congestion routes. For this reason, we propose a new metric which achieve load balancing between the selected routes by taking into account the number of active paths through every node.

In general, the number of links passing over a node is not restricted. In the mean time when the number of links increases, it leads to congestion and contention problem. It causes a high delay, more control overheads and performance degradation due to its node mobility, large queue size and deficiency of bandwidth. To overcome this problem, we introduce a threshold value that limit the number of links passing over a node. This new congestion-avoidance routing scheme is called as Path Efficient Ad-hoc on-demand Multipath Distance Vector (PE-AOMDV) [29] [30] routing protocol.

Consider a MANET with $N$ nodes, whose topology can be described as the interconnection links between $N$ nodes, as well as a connected graph $\mathrm{G}(\mathrm{V}, \mathrm{E})$, where $\mathrm{V}=\{n i, i=1, \ldots, N\}$ is the set of nodes and $\mathrm{E} \subset \mathrm{V} \times$ $\mathrm{V}$ is the set of edges of the graph. Let $\operatorname{Rt}(n i)$ and $R c(n i)$ denote the transmission range and carrier sensing range of node $n i$, respectively. For $n i \in \mathrm{V}$ and $1 \leq i \leq N$ if $n i$ is inside the transmission range of $n j$ as well as $n j$ is also inside the transmission range of $n i$, then the edge eij $\in \mathrm{E}$.

Definition 1: Path Lij denotes a sequence of edges from a source node $n i$ to a destination node $n j$, and $L i j$ includes all successive links from $n i$ to $n j$. All nodes but the source and the destination over a path are called intermediate nodes. If there are $M$ paths from node $n i$ to $n j$, then the multipaths can be represented as $L i j=\{L m i j, 1 \leq$ $m \leq M\}$.

\subsubsection{Multipath Evaluation Based on Link Load}

Based on the network model mentioned previously, the traffic load at node $n i$ can be defined by equation 3.4

$$
T\left(n_{i}\right)=\sum_{k=m}^{M} S_{k} \quad-\mathbf{3 . 4}
$$

where $M$ is the number of paths and $S k$ is the average number of links passed through at node $n i$ over path $L m_{i j}$ which should not exceed the AP threshold value depending on the application in consideration. Let $Q(L i j)$ denote the traffic load on the link between nodes $n i$ and $n j$. Then, the link load can be defined by equation 3.5

$$
Q\left(L_{i j}\right)=\sum_{p=n_{i}}^{n_{j}} T(p) \quad-\mathbf{3 . 5}
$$

From the above evaluation model load-balancing approach that computes the path vacant ratio is proposed for multiple-paths. The path vacant ratio can be used to evaluate the load over multiple paths, which is derived from taking account of load balancing, path load, important paths, and importance of nodes over multiple-paths.

\section{Proposed Path Efficient and Geographical Distance based Ad-hoc on-demand Multipath Distance Vector routing protocol}

The proposed scheme combines the mechanism of GD-AOMDV and PE-AOMDV schemes. The resulting algorithm Path Efficient and Geographical Distance based Ad-hoc on-demand Multipath Distance Vector routing protocol (PE-GD-AOMDV) works more efficiently than AOMDV in video streaming environment. This scheme takes into consideration the factors such as transmission distance, energy and load within the nodes.This scheme prevents the congestion and contention problem by maintaining the number of links passing through the nodes. The number of links that make up a path for transmitting packet will not exceed the threshold value.

In case of exceeding the threshold value the request to transmit packet gets discarded. The scheme also maintains the life time of a node by selection of minimum transmission distance path to balance energy level,mobility lifetime of the network. This scheme implements the maintenance and checking the number of links, energy level and transmission distance for packet transmission simultaneously each time when a request comes to a node to transmit packet through it.

The implementation of the protocol helps in overcoming the traffic overhead while transmitting packet. Also the life time of the nodes is also prolonging maintained. So that data can be transmitted for long time without any congestion and loss. The proposed scheme also performs well with respect to quality of the video and mean opinion score.

\subsection{Route Update Process}

To achieve the objective few modifications are made in the route discovery phase of AOMDV to find the minimal nodal residual energy of each route between any source and destination pair. Each RREQ carry an additional field called $\mathrm{AP}_{\mathrm{th}}$ in order to select load aware path. Structure of routing table entries of AOMDV, PE-GD-AOMDV routing protocols are given in Table 1.

In the source node's routing table the value of Active Path Threshold should be assigned to prolong node lifetime for any sort of communication. In the intermediate nodes, the route update rule of PE-Gd-AOMDV is shown in Algorithm 1 invoked whenever a node receives the route advertisement. In other words, when an intermediate node receives RREQ if the sequence number of just received packet is greater than this node, it compares its remaining battery energy with the Energy Factor Threshold of RREQ of this node if it is less than Energy Factor Threshold of 
RREQ of this node in order to limit the node for further communication in this route.

The set up of reverse routes at the destination node of PE-GD-AOMDV routing protocol is just like in AOMDV routing protocol. When a node i receives a RREQ or RREP, it updates its advertised hop count for a destination $\mathrm{d}$ if its sequence number is less than the sequence number of RREQ or RREP of node $\mathrm{j}$.

Each node maintains route list as shown in Table 1. After finding the minimum distance aware path by using the Algorithm 1 in route discovery phase, the multiple routes between any source and destination pair generated with minimum residual energy nodes for communication. This extends node as well as network lifetime to selecting energy aware nodes.

\begin{tabular}{|l|l|}
\hline \multicolumn{1}{|c|}{ AOMDV } & \multicolumn{1}{c|}{ PE-GD-AOMDV } \\
\hline destination address & destination address \\
sequence number & sequence number \\
advertised hop count & advertised hop count \\
route list & route list \\
$\{($ nexthop1, hopcount1), & $\{($ nexthop1, hopcount1, active path counter, distance), \\
(nexthop2, hopcount2) & (nexthop2, hopcount2 active path counter, distance, Active Path \\
expiration time out & threshold)expiration time out \\
\hline
\end{tabular}

Table 1 Structure of Routing Table Entries of AOMDV, PE-GD-AOMDV routing protocols

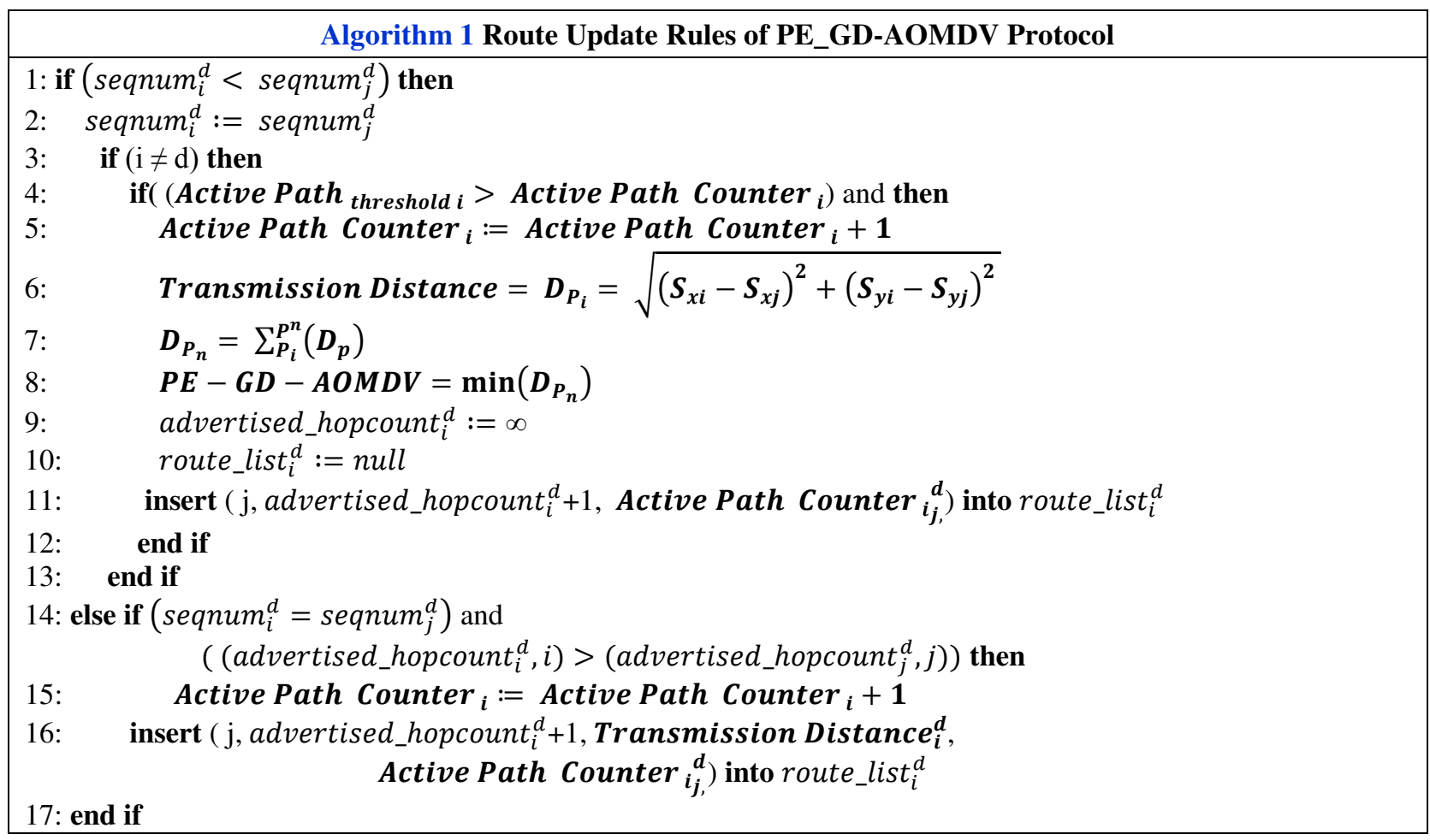

\section{Experimental Classification Results and Analysis}

\subsection{Video Quality Metrics}

Quality of Service (QoS) and Quality of Experience (QoE) are the qualitative measures of the videos have to be delivered over wireless communication networks but QoE reflects the user perception. The most widely used QoE metrics are as follows:

(i) Peak Signal-to-Noise Ratio (PSNR) [31,34] - a traditional objective quality metric used to measure the video quality level based on original and processed video sequences. PSNR of the frames with $\mathrm{M} \times \mathrm{N}$ pixels and 8 bits/sample is defined using Equation 1 as follows: 


$$
P S N R=20 \log _{10}\left[\frac{255}{\sqrt{\frac{1}{M x N} \sum_{i=0}^{M-1 N-1} \sum_{j=0}^{N-1}\|Y s(i . j)-Y d(i, j)\|^{2}}}\right]
$$

(ii) Mean Opinion Score (MOS) [32] - the most widely used subjective quality measure which measures the quality of a system by using "opinion score" in ITU-T Recommendation P.800. Depending on the quality factors to be assessed, MOS can be classified into assessments of listening quality and conversation quality. Possible PSNR to MOS conversion under Absolute Category Rating (ACR) [33] test is shown in Table 2.

MOS can be assessed in terms of listening quality and conversation quality. The listening quality expresses the quality experienced by users when listening to speech and the conversation quality expresses the quality experienced by users when taking part in a conversation.

\begin{tabular}{|l|l|l|}
\hline- & MOS & Category \\
\hline$>37$ & 5 & Excellent \\
\hline $31-37$ & 4 & Good \\
\hline $25-31$ & 3 & Fair \\
\hline $20-25$ & 2 & Poor \\
\hline$<20$ & 1 & Bad \\
\hline
\end{tabular}

Table 2. Possibl e PSNR to MOS conversion under Absolute Category Rating (ACR) test

To evaluate the performance of Path Efficient Geographical Distance based Ad hoc On-demand Multipath Distance Vector (PE-GD-AOMDV) routing on H.264/MPEG-4 AVC video streaming under Distributed Coordination Function (DCF) or Distributed Channel Access (DCA) and Enhanced Distributed Coordination Function (EDCF) or Enhanced Distributed Channel Access (EDCA) using NS2.34 [33,34] and enhanced EvalVid framework. Table 3 shows selected parameters for our simulation design.

\begin{tabular}{|l|l|}
\hline Parameter(s) & Value(s) \\
\hline Simulator & NS-2.34 \\
MAC Types & 802.11 DCF \& 802.11 \\
Packet size & EDCF \\
Hello packet size & 2 Kbyte \\
Other control packet size & 1 Kbyte \\
Frame size & 100 Byte \\
Bits per pixel & 176 x 144 (QCIF) \\
Bit rate & 0.2 \\
Maximum tolerable delay & $56 \mathrm{kbps}$ \\
Averagecodec & $250 \mathrm{~ms}$ \\
power/packet & $500 \mathrm{~mW}$ \\
Average compression & $50-60 \mathrm{~ms}$ \\
delay & \\
\hline
\end{tabular}

Table 3. Simulation parameters

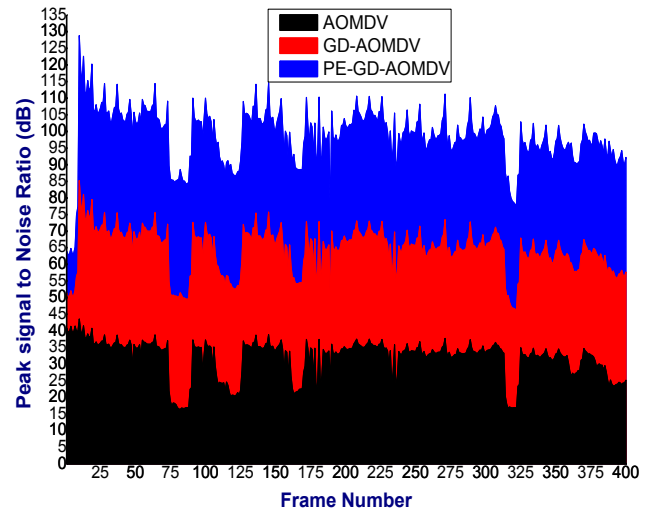

Fig 4. Peak Signal to Noise Ratio (dB) under DCA

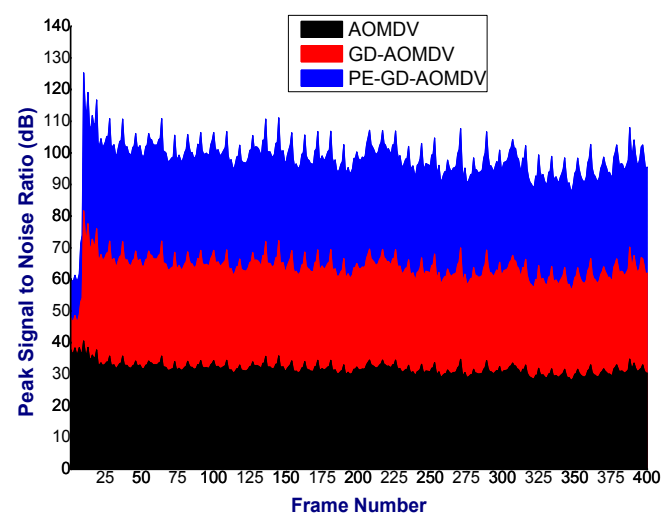

Fig 5. Peak Signal to Noise Ratio (dB) under EDCA

From Figure 4 and Figure 5, it is found that the Peak Signal to Noise Ratio (dB) of PE-GD-AOMDV is better than the GD-AOMDV and AOMDV routing protocols under both DCA and EDCA due to the selection of paths based on minimum transmission distance and active path threshold to limit the load on each path. But AOMDV routing protocol the routes are selected based on only hop count which does not ensure load balance, optimum energy management and edge effect problems.

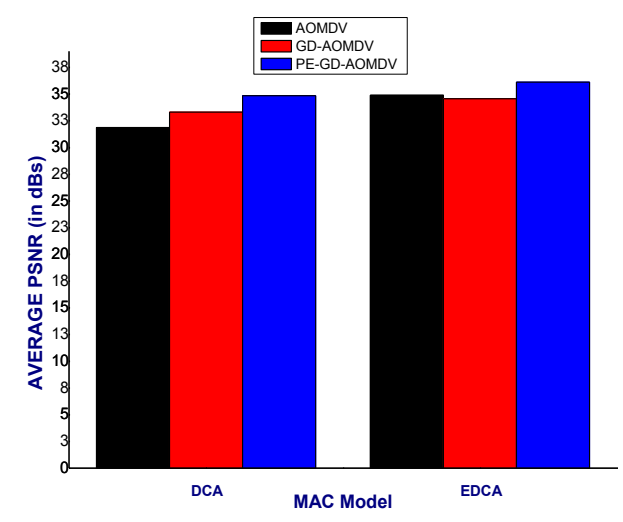

Fig 6. Average PSNR (in $\mathrm{dBs}$ ) 


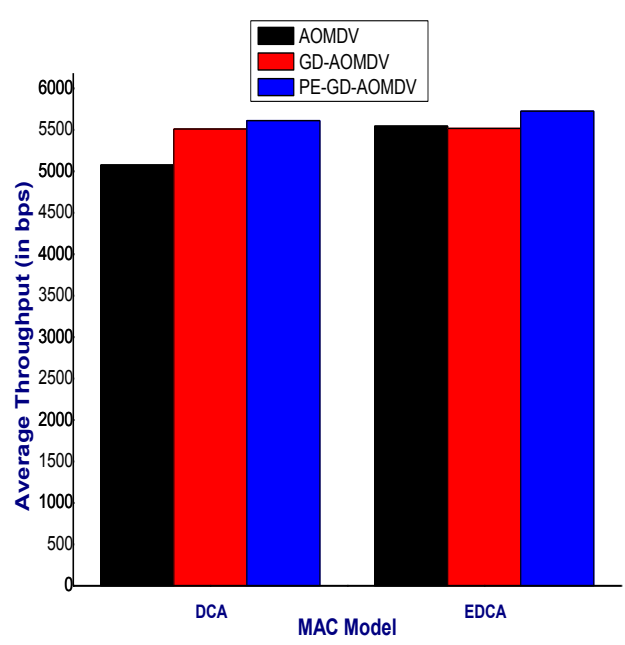

Fig 7. Average Throughput (in bps)

\begin{tabular}{|c|c|c|c|}
\hline $\begin{array}{c}\text { MAC } \\
\text { Model }\end{array}$ & AOMDV & $\begin{array}{c}\text { GD-AOM } \\
\text { DV }\end{array}$ & $\begin{array}{c}\text { PE-GD- } \\
\text { AOMDV }\end{array}$ \\
\hline DCA & 31.8773 & 33.32114 & 34.86213 \\
\hline EDCA & 34.8872 & 34.56666 & 36.12476 \\
\hline
\end{tabular}

Table 4. Average PSNR (in dBs)

\begin{tabular}{|c|c|c|c|}
\hline $\begin{array}{c}\text { MAC } \\
\text { Model }\end{array}$ & $\begin{array}{c}\text { AOM } \\
\text { DV }\end{array}$ & $\begin{array}{c}\text { GD- } \\
\text { AOMDV }\end{array}$ & $\begin{array}{c}\text { PE- } \\
\text { GD- } \\
\text { AOMDV }\end{array}$ \\
\hline DCA & 5078.97872 & 5511.85276 & 5611.87226 \\
\hline EDCA & 5546.88146 & 5520.47853 & 5727.43823 \\
\hline
\end{tabular}

Table 5. Average Throughput (in bps)

From Figure 6, Figure 7 and Table 4, Table 5 , it is also found that the Average PSNR and Throughput of PE-GD-AOMDV is better than the GD-AOMDV and AOMDV routing protocols under both DCA and EDCA due to the selection of paths based on minimum transmission distance and active path threshold to limit the load on each path. But AOMDV uses stale routes and failed to manage energy and congestion problem. Here the proposed PE-GD-AOMDV selects distance and load aware path to reduce the overheads during communication.

\section{Conclusion}

Quality of video delivered over the Internet has been determined in terms of Quality-of-Service (QoS) and Quality-of-Experience (QoE). QoS includes packet delivery ratio, average end-to-end delay, throughput, routing overhead, etc. QoE is a qualitative measure of videos transmitted over Internet. The proposed Path Efficient and Geographical Distance Based Ad hoc
On-demand Multipath Distance Vector (PE-GD-AOMDV) routing protocol is evaluated and the performance on H.264/MPEG-4 AVC video streaming under both Distributed Coordination Function (DCF) and Enhanced Distributed Coordination Function (EDCF) using NS2.34 and my EvalVid framework.

Simulation results showed that the PE-GD-AOMDV performs better than AOMDV in Video streaming environment. In future, the PE-GD-AOMDV will be enhanced to adopt with WiMAX and will also be evaluated by considering Scalable Video Coding (SVC) strategies under cognitive radio or delay/disruption tolerant network environments.

\section{Reference}

[1] W. Wei and Zakhor, Interference aware multipath selection for video streaming in wireless ad hoc networks, IEEE Transactions on Circuits and Systems for Video Technology, 19 (2), 2009, 165-178.

[2] G. Bai, J. Tao and H. Shen , A link-lifetime based dynamic source routing protocol (LTDSR) for multimedia over MANETs, The Chinese Institute of Engineers, 33 (5), 2010, 761-768.

[3] Huifang Chen, Yu Xin and Lei Xie, End-to-end quality adaptation scheme based on QoE prediction for video streaming service in LTE networks, 11th International Symposium on Modeling \& Optimization in Mobile, Ad Hoc \& Wireless Networks (WiOpt), 13-17, 2013, 627-633.

[4] ITU-International Telecommunication Union, Definition of Quality of Experience, Ref: TD 109rev2 (PLEN/12), 2007.

[5] ITU-T Rec. H.264 and ISO/IEC 14496-10 (MPEG4-AVC), Advanced Video Coding for Generic Audiovisual Services,v1, May, 2003; v2, Jan. 2004; v3 (with FRExt), Sept. 2004; v4, 2005.

[6] J. Ostermann, J. Bormans, P. List, D. Marpe, M. Narroschke, F. Pereira, T. Stockhammer, and T. Wedi, Video coding with H.264/AVC: tools, performance, and complexity, Circuits and Systems Magazine, IEEE, 4 (1), 2004, 7-28.

[7] C. H. Ke, C. K. Shieh, W. S. Hwang and A. Ziviani, An Evaluation Framework for More Realistic Simulations of MPEG Video Transmission, Journal of Information Science and Engineering, 24 (2), 2008, 425-440.

[8] J. Klaue, B. Rathke, and A. Wolisz, EvalVid - A framework for video transmission and quality evaluation, Proc. of the International Conference on Modelling Techniques and Tools for Computer Performance Evaluation, 2003, 255-272.

[9] Enhanced EvalVid Framework: http://hpds.ee.ncku.edu.tw/ smallko/ns2/Evalvid_in_ NS2.htm.

[10] NCTU codec, http://megaera.ee.nctu.edu.tw/mpeg.

[11] ffmpeg, http://ffmpeg.sourceforge.net/index.php.

[12] Xvid, http://www.xvid.org/.

[13] tcp-dump, http://www.tcpdump.org.

[14] win-dump, http://windump.polito.it.

[15] Y. Wang and Q. F. Zhu, Error control and 
concealment for video communication: a review, Proceedings of the IEEE, 86, 1998, 974-997.

[16] V.C. Frias and G.D. Delgado et al., Multipath Routing with Layered Coded Video to Provide QoS for Video Streaming over MANET, in IEEE International Conference on Networks, Singapore, 2006, 1-6.

[17] K. Katsaggelos and Dalei Wu et al., Application Centric Routing for Video Streaming over Multihop Wireless Networks, Sensor, Mesh and Adhoc Communications and Networks, Rome, 2009, 385-391.

[18] Sonal Mishra and anil Singh, A Novel Approach for video Transmission in MANET, International Journal of computer Science and Information Technologies, 5(6), 2014, 7270-7275.

[19] Mohammed Halloush, R. Hussein, Al-Zoubi, Zakaria Al-Qudah, and Osameh Alkofahi, "The performance of MANET Routing Protocols for Scalable Video Communication Communications and Network,5, 2013, 119-125.

[20] T. Schierl, C. Hellge, K. Ganger, T. Stockhammer, and T. Wiegand, Multi Source Sssstreaming for Robust Video Transmission in Mobile Ad-Hoc Networks, in IEEE International Conference on Image Processing, Atlanta, GA, USA, Oct. 2006.

[21] Tarek Sheltami, Performance Evaluation of H.264 Protocol in Ad hoc Networks, Journal of Mobile Multimedia, 4( 1), 2008, 59-70.

[22] C.T. Calafate, M.P. Malumbres, J. Oliver, J.C. Cano, and P. Manzoni, QoS Support in MANETs: a Modular Architecture Based on the IEEE 802.11e Technology, IEEE Transactions on Circuits and Systems for Video Technology, 19(5), 2009, 678-692.

[23] Gyeongcheol Lee and Hwangjun Song, Cross layer optimized video streaming based on ieee 802.11 multi-rate over multi-hop mobile ad hoc networks, Mob. Netw. Appl.,15, 2010, 652-663.

[24] A. Valera, W. Seah and S. Rao, Cooperative Packet Caching and Shortest Multipath Routing in Mobile Ad Hoc Networks, Proc. IEEE Infoco, April, 2003.

[25] C.T. Calafate, M.P. Malumbres, J. Oliver, J.C. Cano, and P. Manzoni, QoS Support in MANETs: a Modular Architecture Based on the IEEE 802.11e Technology, IEEE Transactions on Circuits and Systems for Video Technology,19(5), 2009, 678-692.

[26] M.K.Marina and S.R.Das, Ad hoc on-demand multipath distance vector routing", International Journal of Wireless Communications And Mobile Computing, 6( 2), 2001,969-988.

[27] C.E.Perkins and E.M.Royer, Ad hoc on-demand distance vector routing, Proc. IEEE Workshop on Mobile Computing Systems and Applications, New Orleans, LA, 1999, 90-100.

[28] M.Kokilamani and E.Karthikeyan, Location and Mobiltiy Optimized On-demand Geographical Multipath Routing Protocol for MANET, International Journal of Advanced Networking and Apllications,7(4), 2016, 2835-2848.

[29] M. Kokilamani and E.Karthikeyan, A novel optimal path selection strategy in MANET using energy awareness, International Journal of Mobile Network Design and Innovation, 7(3), 2017,129-139.

[30] M. Kokilamani and E.Karthikeyan , An optimal path selection criterion in multipath routing using energy, CSI Transactions on ICT-Springer, DOI 10.1007/s40012-017-0158-8

[31] Speech quality assessment methods, http://www.ntt.co.jp/qos/qoe/eng/technology/sound/i ndex.html

[32] Video quality assessment methods, http://www.ntt.co.jp/qos/qoe/eng/technology/visual/i ndex.html

[33] Kevin Fall, K. Varadhan, The Ns Manual, (University of Southern California, Information Sciences Institute (ISI)) http://www.isi.edu/nsnam/ns/ns-documentation.html.

[34] NS-2 with Wireless and Mobility Extensions, http://monarch.cs.rice.edu/cmu-ns.html

\section{Bibliography}

Dr. M.Kokilamani completed her Ph.D in Computer Science from Bhararhiyar University, Coimbatore,Tamil Nadu, India in 2017 and presently working as Assistant Professor of Computer Science at Kamalam College of Arts and Science, Udumalpet, Tamil Nadu, India. Her research interests include routing protocol design and quality of service (QoS) enhancement of wireless networks such as MANETs. She has published 7 papers in International Journals and Conference proceedings. He has also attended more than 6 conferences at National and International levels.

E. Karthikeyan completed his Ph.D in Computer Science from Gandhigrams University, Dindigul, Tamil Nadu, India in 2008 and presently working as Head \& Assistant Professor of Computer Science at Government Arts College, Udumalpet, Tamil Nadu, India. His research interests include network security and cryptography, MANET routing, congestion control, and advanced networking. He has published 30 papers in international journals and attended more than 15 conferences at National and International levels. He has also published a book entitled Text Book on C: Fundamentals, Data Structures and Programming by PHI. $\mathrm{He}$ is a life member of CSI, CRSI, IASCT etc. and Editor-in-Chief of IJANA 